LA GRANJA:

\section{REVISTA DE}

CIENCIAS DE LA VIDA

pISSN:1390-3799; eISSN:1390-8596

http://doi.org/10.17163/lgr.n34.2021.05
Artículo científico / Scientific paper

Dietas Sostenibles

\title{
INFLUENCIA DE INTERVENCIONES EDUCATIVAS SOBRE NUTRICIÓN Y SOSTENIBILIDAD EN UNIVERSITARIOS ECUATORIANOS RESIDENTES EN HONDURAS
}

\author{
INFLUENCE OF EDUCATIONAL INTERVENTIONS ON NUTRITION AND \\ SUSTAINABILITY IN ECUADORIAN UNIVERSITY STUDENTS RESIDING IN \\ HONDURAS
}

Jean Pierre Enriquez ${ }^{* 1}$ y Adriana Hernández-Santana ${ }^{2}$ (๑)

\author{
${ }^{1}$ Programa de Maestría en Agricultura Tropical Sostenible, Departamento de Posgrado. Escuela Agrícola Panamericana Zamo- \\ rano, Honduras, C.A. \\ ${ }^{2}$ Laboratorio de Nutrición Humana, Departamento de Agroindustria Alimentaria. Escuela Agrícola Panamericana Zamorano, \\ Honduras, C.A.
}

*Autor para correspondencia: jean.enriquez.2018@alumni.zamorano.edu

Manuscrito recibido el 19 de octubre de 2020. Aceptado, tras revisión, el 24 de febrero de 2021. Publicado el 1 de septiembre de 2021.

\section{Resumen}

Los hábitos alimenticios pueden verse comprometidos en la transición a la vida universitaria especialmente en un nuevo país. Además, los patrones alimenticios de los jóvenes universitarios podrían mejorarse mediante diversas estrategias durante el proceso de adaptación a la universidad. Por lo cual, un punto clave lo constituyen la nutrición y la sostenibilidad, cuyo contenido puede ser impartido mediante sesiones educativas en las universidades. El objetivo de este estudio fue evaluar el efecto de intervenciones educativas en la adherencia a la dieta mediterránea (DM) y hábitos alimenticios en estudiantes ecuatorianos de primer año universitario en Honduras, donde la población estudiantil de Ecuador se posiciona en segundo lugar, luego de Honduras. Los patrones alimenticios se evaluaron mediante un cuestionario de adherencia a la DM y uno de conductas alimenticias. Las intervenciones educativas se centraron en los beneficios sobre la sostenibilidad, cultura, ambiente y salud que brinda la DM. El estudio tuvo una fase diagnóstica con la participación del universo de estudiantes $(n=65)$, y una fase de intervenciones donde participaron 32 estudiantes: 18 en el grupo que recibió educación nutricional y 14 en el grupo control. El grupo que recibió intervención educativa mejoró sus conductas alimenticias pasando de poco saludables a moderadamente saludables, sin embargo, la adherencia a la DM no presentó incrementos al final de las intervenciones $(\mathrm{p}>0,05)$. El grupo control no presentó cambios en ninguna evaluación, manteniéndose en conductas poco saludables y una baja adherencia a la DM. Cincuenta por ciento de los estudiantes del grupo con intervención regresaron a Ecuador durante la crisis de COVID-19, mientras que en el grupo control fue el 71,4\%. En conclusión, es importante brindar asesoramiento sobre nutrición y sostenibilidad desde el inicio de los estudios universitarios, ya que los jóvenes migran hacia un país con costumbres totalmente distintas a las de Ecuador e incluso de la Comunidad Andina.

Palabras clave: Dietas sostenibles, educación nutricional, estudiantes ecuatorianos, hábitos alimenticios. 


\begin{abstract}
Eating habits can be compromised in the transition to college life specially in a new country. In addition, the eating patterns of college students could be improved through various strategies during the college adjustment process. A key point is nutrition and sustainability, whose content can be taught through educational sessions at universities. The objective of this study was to evaluate the effect of educational interventions and adherence to the Mediterranean diet (MD) in Ecuadorian first-year university students in Honduras, where the student population of Ecuador ranks second, after Honduras. Eating patterns were evaluated using a questionnaire on adherence to MD and one on eating behaviors. The educational interventions focused on the benefits of sustainability, culture, environment, and health that MD provides. The study had a diagnostic phase with the participation of the universe of students $(\mathrm{n}=65)$, and an intervention phase where 32 students participated: 18 in the group that received nutritional education and 14 in the control group. The group that received educational intervention improved their eating behaviors, going from unhealthy to moderately healthy, however, adherence to MD did not show increases at the end of the interventions ( $p$ $>0.05$ ). The control group did not present changes in any attribute, remaining in unhealthy behaviors and low adherence to MD. Fifty percent of the students in the intervention group returned to Ecuador during the COVID-19 crisis, while in the control group it was $71.4 \%$. In conclusion, it is important to provide advice on nutrition and sustainability from the beginning of university studies, since young people migrate to a country with totally different customs from those of Ecuador and even the Andean Community.
\end{abstract}

Keywords: Eating habits, Ecuadorian students, nutritional education, sustainable diets.

Forma sugerida de citar: Enriquez, J.P. y Hernández-Santana, A. (2021). Influencia de intervenciones educativas sobre nutrición y sostenibilidad en universitarios Ecuatorianos residentes en Honduras). La Granja: Revista de Ciencias de la Vida. Vol. 34(2):80-90. http://doi.org/10. 17163/lgr.n34.2021.05.

IDs Orcid:

Jean Pierre Enriquez: http:/ / orcid.org/0000-0001-6174-6429

Adriana Hernández-Santana: http:/ / orcid.org/0000-0002-7802-3611 


\section{Introducción}

Los cambios en las prácticas agrícolas han incrementado la capacidad mundial de abastecimiento de alimentos de una manera más productiva, brindando mayor diversidad de alimentos y una menor dependencia estacional (Kearney, 2010). Por lo cual, es importante velar por asegurar la estabilidad alimentaria mediante el acceso, utilización y condiciones económicas en los alimentos, lo que guarda una estrecha relación con los principios de la seguridad alimentaria (Anderson, 2019). Además, según la Organización Mundial de la Salud (OMS), las enfermedades no transmisibles (ENT) son la principal causa de muerte en el mundo (WHO, 2014). Por otro lado, un dato alentador indica que en la última década la probabilidad de muerte prematura por ENT se ha reducido $15 \%$ a nivel mundial, siendo los países de altos ingresos quienes exhiben los mayores progresos gracias a la reducción de la mortalidad (WHO, 2015).

Existen patrones de consumo alternativos como los alimentos orgánicos que buscan mejorar la salud y hábitos alimenticios de las personas. Es así como estos alimentos son considerados más saludables, sabrosos y auténticos que la comida tradicional, sin embargo, se catalogan también como más costosos (Bryła, 2016). En este sentido, el precio es una de las principales barreras para las dietas saludables. Por ejemplo, en Galápagos-Ecuador, sus residentes han aumentado el consumo de alimentos procesados y ultra procesados debido a su fácil acceso, disminuyendo así el consumo de productos frescos (Freire y col., 2018).

Así, las dietas sostenibles buscan suplir los requerimientos energéticos mediante alimentos inocuos de alta calidad nutricional, siendo alcanzables, accesibles y culturalmente adecuadas (Dernini y col., 2016). Además, de poseer un impacto ambiental reducido, contribuyen a la seguridad alimentaria y nutricional (SAN) y orientan a la población hacia una vida sana (FAO, 2012; Donini y col., 2016). Entre las dietas sostenibles, figura la dieta mediterránea (DM), siendo presentada como modelo cultural y ecológico de prevención y reducción del riesgo de ENT como enfermedades autoinmunes, cardiovasculares, síndrome metabólico, cáncer y neurodegenerativas (Dussaillant y col., 2016; Cadarso y col., 2017; Gómez, 2018), que pueden traer severas consecuencias en la edad adulta, pero un adecuado estilo de vida especialmente desde los años universitarios o incluso antes pueden ayudar a prevenirlas.

La mayoría de los campus universitarios tienen comedores que ofrecen una variedad de opciones de alimentos, generando comportamientos alimentarios positivos y también no tan adecuados (Abraham, Noriega y Shin, 2018). La transición a la universidad provoca cambios significativos en las opciones dietéticas, generándose incluso desafíos dietéticos. Incluso, las costumbres del colegio pueden ser una determinante para los hábitos alimenticios en la universidad. Sánchez y col. (2018), en un colegio ecuatoriano encontraron que el $90 \%$ de sus estudiantes consumen comida chatarra y snacks durante su receso, apuntando al riesgo de manifestaciones tempranas de enfermedades metabólicas, de alto costo social y económico para la familia y el estado.

Los estudiantes pueden tener un conocimiento competente sobre los requisitos nutricionales. Sin embargo, la transición a la vida universitaria les da más libertad para elegir el tipo y la cantidad de alimentos que comen. Este patrón se genera debido a la inevitable situación de que los estudiantes universitarios se enfrenten a un nuevo entorno para la preparación y planificación de las comidas durante la transición a su vida universitaria (Abraham, Noriega y Shin, 2018). El realizar intervenciones educativas permite que un consumidor se relacione con el concepto de consumo responsable, que implica cierta conciencia y elecciones activas. En Quito, los ciudadanos pueden incidir en su entorno alimentario a través de campañas y organizaciones que promuevan la creación de mercados barriales, abiertos y agroecológicos (Paredes y col., 2019). Sin embargo, una sola medida promocional no es suficiente para desalentar el consumo de alimentos poco saludables.

Actualmente no se han desarrollado estudios sobre los comportamientos alimenticios para universitarios ecuatorianos en Honduras por tal motivo el objetivo del presente estudio fue evaluar el efecto de intervenciones educativas y la adherencia a una dieta saludable y sostenible (dieta mediterránea) en estudiantes ecuatorianos de primer año universitario en Honduras. 


\section{Materiales y Métodos}

\subsection{Diseño del estudio}

Se realizó un estudio analítico de cohorte descriptivo longitudinal entre febrero y junio del 2020 en la Escuela Agrícola Panamericana Zamorano, localizada en Honduras que cuenta con una población estudiantil de alrededor de 29 países principalmente del continente americano, los cuales viven en un internado de enero a diciembre por cuatro años. Los datos presentados en este estudio se derivan de encuestas de conductas alimenticias y adherencia a la DM antes y después de intervenciones educativas nutricionales en la pandemia de COVID-19.

\subsection{Participantes}

Se convocaron 65 estudiantes ecuatorianos para recibir una charla magistral de educación nutricional durante el primer periodo del año académico 2020, antes de que iniciara la pandemia de COVID-19 en América Latina. En la reunión se aprovechó a reclutar de manera voluntaria a dichos estudiantes, previo a socializar el estudio indicando el objetivo, temática y beneficios para el participante e investigador. La participación fue voluntaria, mediante la firma del consentimiento informado. Para este análisis se consideraron únicamente estudiantes ecuatorianos de primer año, debido a que el estudio contó con la primera toma de datos en el segundo mes de clases. Además, se excluyeron a los estudiantes de segundo, tercero y cuarto año, quienes ya tuvieron su etapa crítica de transición al sistema universitario y al ambiente cultural del nuevo país.

\subsection{Fase diagnóstica}

Para contextualizar la situación de los 65 estudiantes ecuatorianos, se realizó la toma de datos de conductas alimenticias y adherencia a la DM. Cuarenta y dos participantes fueron del sexo masculino y 23 del femenino.

\subsection{Fase intervenciones}

Al iniciar el periodo lectivo en el primer año, la universidad aleatoriza equitativamente a los estudiantes por nacionalidad y género en ocho subgrupos. Con este antecedente se procedió a asignar aleatoriamente cuatro de dichos subgrupos para conformar el grupo control y los cuatro restantes al grupo que recibiría intervenciones. Se obtuvo una muestra final de 32 participantes. Se contó con un grupo (n $=18$ ) al cual se le brindó intervenciones de educación nutricional, de los cuales 7 estudiantes fueron del género masculino y 11 del femenino. Se realizaron ocho sesiones de aproximadamente una hora, cuatro de ellas de manera presencial y cuatro de manera virtual debido a la emergencia del COVID19. La temática se centró en las bondades de la DM en los ámbitos de nutrición, salud, sostenibilidad, biodiversidad e impacto ambiental. Las plataformas electrónicas utilizadas para compartir contenido entre el investigador y los participantes fueron redes sociales (Facebook, WhatsApp y Microsoft Teams). El uso de redes sociales incluyó información educativa, mensajes, recordatorios, encuestas e información de eventos relacionados con la DM. Se enfatizó en la adecuada elección de alimentos por los consumidores y sus beneficios a futuro. Por su parte, el grupo control $(n=14)$ no recibió educación nutricional, aquí 6 participantes pertenecieron al género masculino y 8 al femenino.

\subsection{Instrumentos}

Para las conductas alimenticias se utilizó un cuestionario de 31 preguntas de opción múltiple diseñado y validado por Márquez y col. (2014), omitiendo dos preguntas que representan los horarios y personas con quien ingiere los alimentos, entre semana y el fin de semana, debido a que no aplican en el ambiente estudiantil de Zamorano, ya que es un internado con un horario establecido de comidas y el círculo social de los estudiantes son sus propios compañeros, teniendo un número final de 29 preguntas. La temática de las preguntas se centró en los hábitos, comportamientos y gustos alimenticios en escenarios personales, familiares, culturales e institucionales. Las puntuaciones obtenidas de los hábitos alimenticios se agrupan en cuatro categorías: saludables (23-30 puntos), moderadamente saludables (16-22 puntos), poco saludables (8-15 puntos) y muy poco saludables ( $<8$ puntos). También se tomó en consideración la autopercepción de mejora en conductas alimenticias en la pandemia COVID-19.

Para la adherencia a la DM, se aplicó el cuestionario de 14 puntos, utilizado en el estudio PREDIMED (Prevención con Dieta Mediterránea), estudio de intervención nutricional realizado y validado en España, utilizado para evaluar a largo plazo la efi- 
cacia de la DM en la prevención primaria de enfermedades cardiovasculares (Martínez y col., 2015). Dicho cuestionario está compuesto de 14 preguntas directas sobre el consumo de los principales alimentos de la DM: aceite de oliva, frutas, verduras y hortalizas, legumbres, pescado, frutos secos, consumo moderado de vino y de carnes blancas, y escaso consumo de carnes rojas y procesadas. Las puntuaciones obtenidas se agrupan en cuatro categorías de adhesión: alta (12-14 puntos), media (8-11 puntos), baja (5-7 puntos) y muy baja ( $<5$ puntos).

\subsection{Estadística}

Para la fase diagnóstica, los resultados se resumieron utilizando estadísticas descriptivas con medias, porcentajes y desviación estándar. Por su parte, para la fase de intervenciones, se realizó una prueba de comparación de muestras independientes (grupo control y grupo al que se aplicaron intervenciones educativas), con el objetivo de identificar el efecto de las intervenciones educativas en las conductas y adherencia a la DM. Se realizó también un análisis de muestras pareadas mediante la prueba t-student (antes y después en cada grupo) para identificar diferencias entre los efectos de las conductas y adherencia a la DM. Además, se realizó una prueba de McNemar para evaluar la significancia de las distribuciones de adherencia y conductas alimenticias en los dos períodos. Todos los análisis tuvieron un nivel de confianza del $95 \%$, y se utilizaron los programas JAMOVI y Statistical Analysis Software SAS ${ }^{\circledR}$ versión 9.4.

\section{Resultados}

\subsection{Fase diagnóstica}

Para las conductas alimenticias, la media general fue de 14,28 que muestra conductas alimenticias poco saludables. La mayoría (68\%) se ubicaron en conductas poco saludables y muy poco saludables. Además, ninguno de los participantes presentó conductas saludables. En el caso de la adherencia a la DM, se obtuvo una media general de 8,05 indicando una adherencia media. La mayoría de los participantes $(94 \%)$ se ubicaron en adherencia media y baja (Tabla 1).

Tabla 1. Distribución de las conductas alimenticias y adherencia a la DM a través de sus respectivos niveles.

\begin{tabular}{llllll}
\hline Conductas alimenticias & $\mathbf{n}$ & $\boldsymbol{\%}$ & Adherencia a la DM & $\mathbf{n}$ & $\boldsymbol{\%}$ \\
\hline Saludable & 0 & 0 & Alta adherencia & 2 & 3 \\
\hline Moderadamente saludable & 21 & 32 & Adherencia media & 40 & 62 \\
\hline Poco saludable & 43 & 66 & Baja adherencia & 21 & 32 \\
\hline Muy poco saludable & 1 & 2 & Muy baja adherencia & 2 & 3 \\
\hline
\end{tabular}

\subsection{Fase intervenciones}

En el grupo que recibió intervenciones educativas para las conductas alimenticias de la primera toma de datos se observó que un $72 \%$ mostraba conductas poco saludables. Sin embargo, para la segunda toma de datos, el $50 \%$ de los participantes se ubicaron en conductas moderadamente saludables y saludables. En cuanto a la adherencia a la DM, en la primera toma de datos, el $61 \%$ se situaba en adherencia media, manteniéndose en la misma clasificación con un $44 \%$ de los participantes para la segunda toma de datos (Tabla 2). En este grupo, el $50 \%$ de los estudiantes se encontraban en el campus durante la pandemia y el $50 \%$ en Ecuador.
En cuanto al grupo control, para la primera toma de datos, el $64 \%$ de los participantes se situó en conductas poco saludables, manteniéndose en la misma clasificación en la segunda toma de datos con $43 \%$ de los participantes. En la adherencia a la DM, en la primera toma de datos el $64 \%$ de las observaciones pertenecían a adherencia media y el $36 \%$ a baja adherencia. Para la segunda toma la adherencia media se reflejó en el 50\% de los participantes (Tabla 2). En este grupo, el 28,6\% de los estudiantes se encontraban en el campus durante la pandemia y el $71,4 \%$ en Ecuador.

Según la prueba de McNemar, los cambios en las distribuciones de las conductas alimenticias en los dos periodos resultaron significativos en el grupo 
que recibió intervenciones educativas, en conductas poco saludables $(\mathrm{p}=0,046)$. Para la adherencia a la $\mathrm{DM}$, únicamente resulto significativo en el grupo control, en adherencia media $(p=0,025)$.

Tabla 2. Distribución del grupo con intervenciones y el control en las dos tomas de datos, con prueba McNemar.

\begin{tabular}{|c|c|c|c|c|c|c|c|c|c|c|}
\hline \multirow{3}{*}{ Conductas alimenticias } & \multicolumn{5}{|c|}{ Grupo intervención } & \multicolumn{5}{|c|}{ Grupo control } \\
\hline & \multicolumn{2}{|c|}{ Antes } & \multicolumn{2}{|c|}{ Después } & \multirow[b]{2}{*}{$p$} & \multicolumn{2}{|c|}{ Antes } & \multicolumn{2}{|c|}{ Después } & \multirow{2}{*}{$p$} \\
\hline & $\mathrm{n}$ & $\%$ & $\mathrm{n}$ & $\%$ & & $\mathrm{n}$ & $\%$ & $\mathrm{n}$ & $\%$ & \\
\hline Saludable & 0 & 0 & 1 & 6 & - & 0 & 0 & 1 & 7 & - \\
\hline $\begin{array}{l}\text { Moderadamente } \\
\text { saludable }\end{array}$ & 5 & 28 & 8 & 44 & 0,083 & 5 & 36 & 6 & 43 & 0,317 \\
\hline $\begin{array}{l}\text { Poco } \\
\text { saludable }\end{array}$ & 13 & 72 & 9 & 50 & 0,046 & 9 & 64 & 6 & 43 & 0,083 \\
\hline $\begin{array}{l}\text { Muy poco } \\
\text { saludable }\end{array}$ & 0 & 0 & 0 & 0 & - & 0 & 0 & 1 & 7 & - \\
\hline \multicolumn{11}{|l|}{ Adherencia a la DM } \\
\hline $\begin{array}{l}\text { Alta } \\
\text { adherencia }\end{array}$ & 1 & 6 & 0 & 0 & - & 0 & 0 & 1 & 7 & - \\
\hline $\begin{array}{l}\text { Adherencia } \\
\text { media }\end{array}$ & 11 & 61 & 8 & 44 & 0,083 & 9 & 64 & 4 & 29 & 0,025 \\
\hline $\begin{array}{l}\text { Baja } \\
\text { adherencia }\end{array}$ & 6 & 33 & 8 & 44 & 0,157 & 5 & 36 & 7 & 50 & 0,157 \\
\hline $\begin{array}{l}\text { Muy baja } \\
\text { adherencia }\end{array}$ & 0 & 0 & 2 & 11 & - & 0 & 0 & 2 & 14 & - \\
\hline
\end{tabular}

Aquellos espacios que no poseen un valor numérico para la probabilidad se debe a que en uno de los dos periodos se tiene cero observaciones.

De la Tabla 3 se destaca que el grupo que recibió intervenciones educativas, la media de las conductas alimenticias incrementó de 13,89 (poco saludable) a 16 (moderadamente saludable). Para el grupo control, en la primera toma de datos se reportó una media general de 14,79 en conductas alimenticias, situándose en conductas poco saludables. Sin embargo, en la segunda toma de datos, la media de las conductas alimenticias fue de 14,71.

De acuerdo a la prueba $t$ de muestras pareadas únicamente existió significancia para el grupo con intervenciones educativas en las conductas alimenticias, donde existió diferencia entre las conductas alimenticias posterior a dichas intervenciones $(\mathrm{p}<$ $0,001)$. Para el análisis de muestras independientes, las conductas y la adherencia a la DM no fueron significativamente diferentes en ningún período $(p>$
$0,05)$. En cuanto a la Tabla 4, se destaca lo siguiente: en el grupo con intervenciones, inicialmente, la media general de la adherencia se situó en 8,5; posteriormente se situó en 7,06 para la segunda toma de datos. Para el grupo control, la media inicial fue de 7,93 situándose en baja adherencia, ya en la segunda toma la media se situó en 7 con baja adherencia.

De acuerdo a la prueba $\mathrm{t}$ de muestras pareadas únicamente existió significancia para el grupo con intervenciones educativas, donde existió diferencia entre la adherencia inicial con la posterior $(p=0,010)$. Para el análisis de muestras independientes, las conductas y la adherencia a la DM no fueron significativamente diferentes en ningún período $(\mathrm{p}>0,05)$. 
Tabla 3. Conductas alimenticias de los dos grupos en los dos periodos de tiempo con muestras pareadas e independientes.

\begin{tabular}{cccccc}
\hline & \multicolumn{2}{c}{ Intervención } & \multicolumn{2}{c}{ Control } & \multirow{2}{*}{$\begin{array}{c}p \text { Muestras } \\
\text { independientes }\end{array}$} \\
\cline { 2 - 4 } & Media & D.E. & Media & D.E. & \\
\hline $\begin{array}{c}\text { Conductas } \\
\text { alimenticias } \\
\text { antes }\end{array}$ & 13,89 & 3,14 & 14,79 & 3,45 & 0,449 \\
$\begin{array}{c}\text { Conductas } \\
\text { alimenticias } \\
\text { después }\end{array}$ & 16,00 & 3,34 & 14,71 & 5,34 & 0,410 \\
\cline { 1 - 4 } & Student's t & $p$ & Student's t & $p$ & \\
\cline { 1 - 4 } $\begin{array}{c}\text { Muestras } \\
\text { pareadas }\end{array}$ & $-4,421$ & $<0,001$ & 0,053 & 0,959 & \\
\hline
\end{tabular}

Tabla 4. Adherencia a la Dieta Mediterránea (DM) de los dos grupos en los dos periodos de tiempo, con muestras pareadas e independientes.

\begin{tabular}{cccccc}
\hline & \multicolumn{2}{c}{ Intervención } & \multicolumn{2}{c}{ Control } & \multirow{2}{*}{$\begin{array}{c}p \text { Muestras } \\
\text { independientes }\end{array}$} \\
\cline { 2 - 4 } & Media & D.E. & Media & D.E. & \\
\hline $\begin{array}{c}\text { Adherencia a } \\
\text { la DM antes }\end{array}$ & 8,50 & 1,76 & 7,93 & 2,09 & 0,407 \\
$\begin{array}{c}\text { Adherencia a } \\
\text { la DM después }\end{array}$ & 7,06 & 1,80 & 7,00 & 2,60 & 0,943 \\
\cline { 1 - 4 } & Student's t & $p$ & Student's t & $p$ & \\
\cline { 1 - 4 } $\begin{array}{c}\text { Muestras } \\
\text { pareadas }\end{array}$ & 2,89 & 0,010 & 1,447 & 0,172 & \\
\hline
\end{tabular}

\section{Discusión}

Las conductas alimenticias poco saludables del grupo total de estudiantes ecuatorianos $(n=65)$, son un indicador preocupante ya que esto podría desencadenar problemas de sobrepeso, obesidad o incluso enfermedades crónicas. En este sentido, Racette y col. (2005), mencionan que los mayores incrementos en el sobrepeso y la obesidad ocurren en personas entre 18 y 29 años, rango de edad de un estudiante universitario. Además, la mayoría de los universitarios al dejar el hogar de sus padres se adaptan a cambios sociales, ambientales, y experimentan nuevas responsabilidades financieras (Das y Evans, 2014). Lo cual empeoraría las conductas poco saludables ya manifestadas.

Los estudiantes universitarios pueden representar un grupo con alto riesgo de desarrollar conductas alimentarias anormales y ejercicio compulsivo (Guidi y col., 2009). Además, se encuentran destacando como barreras comunes de una alimentación saludable, las limitaciones de tiempo, refrigerios poco saludables, alimentos prácticos ricos en calorías, estrés, altos precios de los alimentos saludables y el fácil acceso a la comida chatarra (Sogari y col., 2018). En general los universitarios se ven afectados por barreras relacionadas con la disponibilidad de tiempo, barreras ambientales como la falta de opciones de comidas baratas, sabrosas y saludables en el comedor de la universidad (Hilger y Diehl, 2019). Es aquí cuando una intervención oportuna de educación nutricional puede ser un punto clave para mejorar los estilos de vida de los jóvenes universitarios.

Se ha identificado que las intervenciones educativas lograron mejorar los hábitos alimenticios pasando de poco saludables a moderadamente saludables $(p<0,001)$ en el grupo que completó el curso capacitación. Esto concuerda con lo encontrado por Hekler, Gardner y Robinson (2010), donde aquellos estudiantes que llevaron un curso de Alimentos y Sociedad incrementaron su alimentación saluda- 
ble, obteniendo mejoras en el consumo de vegetales disminuyendo también la ingesta de lácteos ricos en grasas. Además, Boyle y LaRose (2009), descubrieron que los factores intra e interpersonales, así como ambientales, afectan la actividad física y los hábitos alimenticios de los estudiantes universitarios. Boucher, Gagné y Côté (2015), encontraron que las intervenciones educativas incrementaron el consumo de frutas y verduras en estudiantes, haciendo hincapié en la importancia de desarrollar intervenciones adaptadas a los estudiantes universitarios para promover comportamientos saludables. Por otro lado, quienes perciben que la intoxicación alimentaria es una amenaza personal tienden a comer menos alimentos peligrosos (Byrd y col., 2008).

El cambio de $22 \%$ (del 72 al 50\%) de estudiantes con conductas alimenticias poco saludables ( $\mathrm{p}$ $=0,046)$ muestra la reciprocidad de los participantes por poner en práctica la información obtenida, contrario al grupo control en donde a pesar de la disminución del 64 a $43 \%$ no existió diferencia significativa $(\mathrm{p}=0,083)$. Esto concuerda con lo encontrado por Reed y col. (2011), en el que 10 al 19\% de los universitarios participantes del estudio modificaron sus elecciones alimenticias posterior a recibir la información de la intervención.

Una encuesta sobre la efectividad de una aplicación para teléfonos inteligentes en promoción de la salud para estudiantes universitarios mostró que muchos estudiantes no tenían buena salud y no presentaban comportamientos saludables, pese a declarar que la aplicación era útil, beneficiosa y aumentaba la autoconciencia (Miller, Chandler y Mouttapa, 2015). Lo cual concuerda con nuestro estudio en donde a pesar de completar el curso de educación nutricional con una buena participación, no todos los estudiantes mejoraron sus hábitos alimenticios. Una posibilidad a esto es que, frente a la pandemia, el estrés relacionado con el encierro haya provocado alteraciones respecto a los atracones y restricciones dietéticas (Flaudias y col., 2020).

El 50 y 71,4\% de los estudiantes del grupo con intervenciones y control, respectivamente, regresaron a Ecuador por la pandemia, lo que podría ser una de las causas que se obtengan resultados positivos en la mejora de hábitos alimenticios, ya que contaban con el apoyo familiar para aplicar en conjunto los conocimientos adquiridos. Esto concorda- ría con lo encontrado en un estudio etnográfico que examinó las trayectorias universitarias de los estudiantes rurales de primera generación, en donde debido a la importancia de las políticas y prácticas familiares, institucionales, estatales y federales, se debe involucrar a las familias y replicar los modelos de apoyo familiar (Beasley, 2016).

Los intentos de cambiar las dietas de la población a menudo adoptan enfoques muy individualistas, que pueden pasar por alto factores estructurales que influyen en el acceso y la disponibilidad de opciones de alimentos saludables (VanHeuvelen y VanHeuvelen, 2019). La mayoría de las personas están de acuerdo en que los hábitos alimenticios de la población no son saludables, sin embargo, perciben que comen de manera más saludable que otras personas de su entorno (Sproesser y col., 2015). Por lo tanto, se necesitan esfuerzos en los campus universitarios para promover estilos de vida saludables entre su población estudiantil durante los años universitarios (Gropper y col., 2012).

Al evaluar la adherencia a la DM del grupo completo de estudiantes ecuatorianos en fase diagnóstica, se obtuvo en promedio 8,05 puntos, posicionando al grupo en adherencia media. Sin embargo, una vez concluidas las intervenciones con los 18 participantes de este grupo se tuvo una disminución significativa ( $p=0,01)$, en esta adherencia, disminuyendo a baja adherencia, denotando niveles mayores al $50 \%$ en los dos grupos con baja y muy baja adherencia. Esto concuerda con Míguez y col. (2013), quien encontró que el $90 \%$ de los estudiantes universitarios necesita modificar sus hábitos alimenticios para adecuarse a este patrón dietético. Además, un estudio realizado en una población universitaria española también concuerda con nuestro estudio, donde el 96,1\% de los sujetos necesita mejorar la calidad de su dieta y únicamente el 5,3\% de los estudiantes consiguieron una alta adherencia a la dieta mediterránea (García y col., 2014).

Un estudio sobre conocimiento nutricional en Italia muestra que este se asoció significativamente con una mayor adherencia a la DM independientemente de los factores socioeconómicos (Bonaccio y col., 2013). Lo anterior contrasta con esta investigación, en donde los factores socioeconómicos, especialmente por el COVID-19, pudieron haber limitado la disponibilidad de ciertos alimentos en los 
estudiantes que se encontraban en Ecuador. Navarro y col. (2014), encontraron que los estudiantes que vivían en la casa de sus padres tuvieron un alto porcentaje de adherencia, el cual fue significativamente mayor al de quienes vivían en apartamentos o residencias estudiantiles. Además, la pandemia amenaza a millones de personas que viven o corren riesgo de desarrollar inseguridad alimentaria (Paslakis, Dimitropoulos y Katzman, 2020), situación que pudo presentarse en ambos grupos debido al acceso limitado a ciertos alimentos en todos los países.

Un estudio que buscaba determinar la adherencia a la dieta mediterránea de una población universitaria y analizar varios factores que pueden condicionar su calidad nutricional encontró que el 9,5\% de los universitarios tenía un índice de adherencia bajo, el $62,1 \%$ intermedio y el $28,4 \%$ alto (Durá y Castroviejo, 2011), que concuerda con nuestras distribuciones de la primera toma de datos. Por lo tanto, resulta necesario asegurar que la seguridad alimentaria, las actitudes y comportamientos alimentarios saludables sean una prioridad global para poder garantizar la salud de la población, especialmente frente a la pandemia (Paslakis, Dimitropoulos y Katzman, 2020).

\section{Conclusiones}

Este estudio indica una mayor tendencia en la mejora de las conductas alimenticias al realizarse intervenciones educativas en universitarios. Por lo tanto, por medio de estos programas se pueden disminuir los malos hábitos alimenticios que se generan en la transición del hogar hacia la vida universitaria en un nuevo país. Sin embargo, la adherencia a un patrón dietético saludable y sostenible tuvo una disminución que pudo haberse visto comprometido por los problemas de accesibilidad a ciertos alimentos como consecuencia de la pandemia.

Es necesario generar políticas y programas de apoyo a los estudiantes para fortalecer sus conocimientos sobre nutrición y sostenibilidad. Estas nociones deben ser capaces de generar un factor de impacto en los estudiantes que les permita mejorar sus hábitos alimenticios y adoptar patrones dietéticos que beneficien la salud humana y ambiental. Además, es importante dar este asesoramiento desde el inicio de los estudios universitarios, ya que los jóvenes migran hacia países con costumbres totalmente distintas a su país natal, e incluso de la Comunidad Andina. La mejora en los hábitos alimenticios en tiempos de pandemia en los estudiantes ecuatorianos refleja que es necesario desarrollar programas institucionales que faciliten su proceso de transición a la universidad.

\section{Agradecimientos}

Al programa de Maestría en Agricultura Tropical Sostenible (MATS) de Zamorano y al Instituto de Tecnología en Cuidado de la Salud (ITHC) por el apoyo con los materiales de la investigación. De manera muy especial agradecemos a todos los participantes del estudio.

\section{Referencias}

Abraham, S., B. Noriega y J. Shin (2018). «College students eating habits and knowledge of nutritional requirements». En: Journal of Nutrition and Human Health 2.1, 13-17. Online: https: / / bit.ly / 2RgCFOO.

Anderson, J. (2019). «Concepts of Stability in Food Security». En: Encyclopedia of Food Security and Sustainability. Elsevier, 8-15. Online: https:/ / bit. ly/3xkh4FX.

Beasley, E. (2016). «Country Roads Take Me ... ?: A Cultural Analysis of College Pathways among Rural, First-Generation Students». En: Paradoxes of the Democratization of Higher Education (Research in Social Problems and Public Policy). Vol. 22. Emerald Group Publishing Limited, 127-163. Online: https:/ / bit.ly/3aTGyQY.

Bonaccio, M. y col. (2013). «Nutrition knowledge is associated with higher adherence to Mediterranean diet and lower prevalence of obesity. Results from the Moli-sani study». En: Appetite 68, 139-146. Online: https://bit.ly/3uPmJ4H.

Boucher, D., C. Gagné y F. Côté (2015). «Effect of an intervention mapping approach to promote the consumption of fruits and vegetables among young adults in junior college: A quasiexperimental study». En: Psychology \& Health 30.11, 1306-1325. Online: https : / / bit . ly / 32m7jZj. 
Boyle, J. y N. LaRose (2009). "Personal beliefs, the environment and college students' exercise and eating behaviors». En: American Journal of Health Studies 23.4, 195-200. Online: https: / / bit.ly / 3giRhYm.

Bryła, P. (2016). «Organic food consumption in Poland: Motives and barriers». En: Appetite 105, 737-746. Online: https://bit.ly/2SaKzK3.

Byrd, C. y col. (2008). «Risky Eating Behaviors of Young Adults - Implications for Food Safety Education». En: Journal of the American Dietetic Association 108.3, 549-552. Online: https: / / bit. ly/32emzHU.

Cadarso, A. y col. (2017). «Calidad de vida relacionada con la salud y su relación con la adherencia a la dieta mediterránea y la actividad física en universitarios de Galicia (Health related to quality of life and their relationship with adherenceto the mediterranean diet and physical activity at the university in Galicia)». En: Nutrición Clínica y Dietética Hospitalaria 37.2, 42-49. Online: https://bit.ly/3x0SY2y.

Das, M. y E. Evans (2014). «Understanding Weight Management Perceptions in First-Year College Students Using the Health Belief Model». En: Journal of American College Health 62.7, 488-497.Online: https://bit.ly/3aiX6RR<.

Dernini, S. y col. (2016). «Med Diet 4.0: the Mediterranean diet with four sustainable benefits». En: Public Health Nutrition 20.7, 1322-1330. Online: https://bit.ly/3gghSp6.

Donini, L. y col. (2016). «A Consensus Proposal for Nutritional Indicators to Assess the Sustainability of a Healthy Diet: The Mediterranean Diet as a Case Study». En: Frontiers in nutrition 3.37, 1-14. Online: https://bit.ly/3uZqogy.

Durá, T. y A. Castroviejo (2011). «Adherence to a Mediterranean diet in a college population». En: Nutrición Hospitalaria 26.3, 602-608. Online: https:/ / bit.ly/3aimili.

Dussaillant, C. y col. (2016). «Evidencia actual sobre los beneficios de la dieta mediterránea en salud (Current evidence on the health benefits of the Mediterranean diet)». En: Revista médica de Chile 144.8, 1044-1052. Online: https: / / bit.ly / $3 \mathrm{mRgcnm}$.

FAO (2012). Sustainable Diets and Biodiversity. 1st. Rome. Online: https:/ / bit.ly/3nEQv9Q.

Flaudias, V. y col. (2020). "COVID-19 pandemic lockdown and problematic eating behaviors in a student population». En: Journal of Behavioral
Addictions 9.3, 826-835. Online: https: / / bit.ly / $3 \mathrm{mRloHS}$.

Freire, W. y col. (2018). «Overweight, obesity, and food consumption in Galapagos, Ecuador: a window on the world». En: Globalization and Health 14.93, 1-9. Online: https: / / bit.ly/3gg5plc.

García, M. y col. (2014). «Adherence to Mediterranean diet in a Spanish university population». En: Appetite 78, 156-164. Online: https:/ / bit.ly / $3 \mathrm{dn} 6 \mathrm{~s} 0 \mathrm{~S}$.

Gómez, R. (2018). «Revisión sobre el aceite de oliva: historia, impacto mediático y sus aplicaciones médicas en la dieta mediterránea (Review of olive oil: history, media impact and its medical applications in the Mediterranean diet)». En: Revista Colombiana de Endocrinología, Diabetes $\mathcal{E}$ Metabolismo 5.4, 26-31. Online: https: / / bit.ly / 3 tqnPU5.

Gropper, S. y col. (2012). «Weight and Body Composition Changes during the First Three Years of College». En: Journal of Obesity 2012, 1-6. Online: https://bit.ly/3e8N86X.

Guidi, J. y col. (2009). "The prevalence of compulsive eating and exercise among college students: An exploratory study». En: Psychiatry Research 165.1-2, 154-162. Online: https: / / bit.ly / 2Qu3n6g.

Hekler, E., C. Gardner y T. Robinson (2010). «Effects of a College Course About Food and Society on Students Eating Behaviors». En: American Journal of Preventive Medicine 38.5, 543-547. Online: https://bit.ly/3upMxo0.

Hilger, J. y K. Diehl (2019). «'Oh God, I Have to Eat Something, But Where Can I Get Something Quickly?' A Qualitative Interview Study on Barriers to Healthy Eating among University Students in Germany». En: Nutrients 11.10, 2440. Online: https:/ / bit.ly/3ajn6N8.

Kearney, J. (2010). «Food consumption trends and drivers». En: Philosophical transactions of the royal society B: biological sciences 365.1554, 2793-2807. Online: https:/ / bit.ly/3gXjKTV.

Márquez, Y. y col. (2014). «Diseño y validación de un cuestionario para evaluar el comportamiento alimentario en estudiantes mexicanos del área de la salud (Design and validation of a questionnaire to evaluate eating behavior in Mexican students in the health area)». En: Nutrición Hospitalaria 30.1, 153-164. Online: https: / / bit.ly / $3 \times 5 \mathrm{aEtU}$. 
Martínez, M. y col. (2015). «Benefits of the Mediterranean diet: insights from the PREDIMED study». En: Progress in Cardiovascular Diseases 58.1, 50-60. Online: https:/ / bit.ly/3ueZBfY.

Míguez, M. y col. (2013). "Variations of the diet of Galician university students (Ourense Campus) in relation to the pattern of the cardioprotective Mediterranean diet)». En: Nutrición Hospitalaria 28.6, 2099-2106. Online: https:/ / bit.ly/3dpiPtx.

Miller, T., L. Chandler y M. Mouttapa (2015). «A needs assessment, development, and formative evaluation of a health promotion smartphone application for college students». En: American Journal of Health Education 46.4, 207-215. Online: https:/ / bit.ly/2QOzQ7R.

Navarro, I. y col. (2014). "Adherence to the Mediterranean diet by nursing students of Murcia (Spain)». En: Nutrición Hospitalaria 30.1, 165-172. Online: https://bit.ly/3ebOpv1.

Paredes, M. y col. (2019). "Assessing responsible food consumption in three Ecuadorian city regions». En: Sustainable Food System Assessment. Routledge. Online: https:/ /bit.ly/3vATWRF.

Paslakis, G., G. Dimitropoulos y G. Katzman (2020). "A call to action to address COVID-19 induced global food insecurity to prevent hunger, malnutrition, and eating pathology». En: Nutrition Reviews 79.1, 114-116. Online: https: / / bit . ly / 3vA7LQr.

Racette, S. y col. (2005). «Weight Changes, Exercise, and Dietary Patterns During Freshman and Sophomore Years of College». En: Journal of Ame- rican College Health 53.6, 245-251. Online: https: / / bit.ly/3aW2JG5.

Reed, J. y col. (2011). «Using "Point of Decision Messages to Intervene on College Students Eating Behaviors». En: American Journal of Health Promotion 25.5, 298-300. Online: https: / / bit.ly / 3tsM6sB.

Sánchez, M. y col. (2018). «Evaluación del estado nutricional de adolescentes en una Unidad Educativa de Ecuador (Evaluation of the nutritional status of adolescents in an Educational Unit of Ecuador)». En: Ciencia Unemi 10.25, 1-12. Online: https://bit.ly/3v2v7h9.

Sogari, G. y col. (2018). «College Students and Eating Habits: A Study Using An Ecological Model for Healthy Behavior». En: Nutrients 10.12, 1823. Online: https://bit.ly/2Q4eL9i.

Sproesser, G. y col. (2015). «I Eat Healthier Than You: Differences in Healthy and Unhealthy Food Choices for Oneself and for Others». En: $\mathrm{Nu}$ trients 7.6, 4638-4660. Online: https: / / bit.ly / 2QbSUN1.

VanHeuvelen, T. y J. VanHeuvelen (2019). «The (Economic) Development of Healthy Eating Habits: Gender, nutrition, and health outcomes in 31 countries». En: Sociology of Development 5.1, 91-113. Online: https:/ / bit.ly/2RHZBqC.

WHO (2014). Global Status Report of noncommunicable diseases 2014. Online: https:/ / bit.ly/3akfc60.

- (2015). Health in 2015: from MDGs, Millennium Development Goals to SDGs, Sustainable Development Goals. Online: https://bit.ly/2P20PvU. 